\title{
Hacia la Configuración de un Currículo ESP1 para Profesionales Universitarios de los Negocios Internacionales en Honduras
}

\begin{abstract}
José Alexis Espino ${ }^{1}$ y Jean Noel Cooman ${ }^{1}$
${ }^{1}$ Departamento de Lenguas Extranjeras Universidad Nacional Autónoma de Honduras, Honduras | jose.espino@unah.edu.hn; jean.cooman@unah.edu.hn | https://orcid.org/00000002-9749-0880; https://orcid.org/0000-0000-0000-0000

Resumen. Este artículo discute las implicaciones para la creación y gestión de programas eficaces de ESP (Inglés por Objetivos Específicos). Un vasto estudio de corte cualitativo e interdisciplinario, comprendido entre los años 2017 - 2019, se llevó a cabo en un Centro Regional de la Universidad Nacional Autónoma de Honduras. Se estableció como objetivo primordial realizar un profundo diagnóstico de necesidades comunicativo-lingüísticas académicas y profesionales en lo que respecta a aptitudes de comprensión y expresión en inglés de los estudiantes y egresados de las carreras especializadas en negocios internacionales, principalmente, de dicho centro universitario.

Mediante la ejecución, con cuatro poblaciones participantes, de entrevistas individuales y grupales, así como con la técnica de la observación participante, se compiló un corpus extenso de datos, para cuyo análisis e interpretación intensiva se seleccionó pertinentemente, la técnica y el procedimiento meticuloso del análisis del discurso.
\end{abstract}

Palabras claves: ESP (English for specific purposes); ESP para negocios internacionales; Necesidades comunicativo-lingüísticas; Estilos-estrategias de aprendizaje.

\begin{abstract}
Designing ESP Curricula for International Business University Majors in Honduras
Summary. This article discusses the implications for the creation and management of effective ESP programs (English for Specific Purposes). A vast qualitative and interdisciplinary study was carried out between the years 2017 - 2019 in a Regional Center of the National Autonomous University of Honduras. The objective was to carry out an intensive diagnosis of academic and professional communicative-linguistic needs of comprehension and expression skills in English of the students and graduates of careers specialized in international business, mainly, in that university center.

Through the implementation, with four participating groups, of individual and group interviews and the participant observation technique, as well, an extensive body of data was compiled, for the analysis and deep interpretation of which the technique of discourse analysis was appropriately selected and meticulously practiced.
\end{abstract}

Keywords: ESP (English for specific purposes); ESP for international business; Learning styles and strategies; Communication-linguistic needs.

\section{Introducción}

Dentro de un mercado cada vez más globalizado, se impone más allá de alguna duda, el uso del inglés como "lingua franca" (Bracaj, 2014), y en este sentido, la enseñanza del inglés como lengua extranjera debe seguir un procedimiento riguroso para el análisis de necesidades y el desarrollo curricular (Basturkmen, 2010). Abordar la brecha entre el dominio esperado de la lengua y el dominio lingüístico actual de los estudiantes, con sus "deficiencias", "deseos" y "necesidades" representa la función principal de un programa de ESP y de los profesionales calificados (Hutchinson \& Waters, 1987).

Consiguientemente, se inició la presente investigación que determinó las bases teóricas y metodológicas para la elaboración de una propuesta curricular con miras a la gestión de un programa de inglés por objetivos específicos para las Carreras de Administración de Empresas y Comercio Internacional del Centro Universitario Regional Nororiental (CURNO) de la Universidad Nacional Autónoma de Honduras (UNAH).

\footnotetext{
${ }^{1}$ ESP: English for Specific Purposes (Inglés por Objetivos Específicos) (Hutchinson \& Waters, 1987).
} 
Dicho proyecto de investigación pretendió establecer un programa más intensivo que responda directamente a las necesidades comunicativas lingüísticas y las necesidades interculturales específicas de los estudiantes y egresados del rubro de los negocios de esta Universidad, que los lleve a adquirir mejores competencias en inglés hacia un mejor aprovechamiento académico y profesional en su área específica. Esto permitirá simultáneamente el avance socioeconómico de las familias de la comunidad, y paralelamente brinda una respuesta a la demanda de contratación de profesionales capacitados de las cuantiosas empresas nacionales e internacionales de la zona, dedicadas al rubro en mención.

\section{Marco contextual}

Honduras, país centroamericano de nueve millones de habitantes, es como la gran mayoría de los países del continente, una nación multilingüe y multicultural, en la cual el $97 \%$ de la población tiene el español como primera lengua y como lengua principal de enseñanza. Ante esta realidad, la existencia y supervivencia de otras lenguas vernáculas y minoritarias, como el garífuna, el miskito, el pech, el tahwaka, el tol, y una variante de inglés criollo se ven considerablemente comprometidas. Al mismo tiempo, esta fuerte hegemonía del español se combina con la introducción en el sistema de educación pública, de la única lengua extranjera obligatoria, el inglés. En cuanto al uso del inglés en el país, esta lengua cuenta con una presencia muy importante en más de 300 escuelas bilingües, sobre todo en las dos grandes ciudades Tegucigalpa y San Pedro Sula. También es manejado como lengua extranjera por cerca del $10 \%$ de la población hispanohablante del país, principalmente a nivel de comprensión.

La UNAH, espacio donde se ha realizado la investigación, cuenta con una población de casi 90,000 estudiantes, dividida entre la sede en Ciudad Universitaria (CU) en Tegucigalpa, capital del país de 1.3 millones de habitantes, y ocho centros regionales. Dentro de esta universidad pública, es el Departamento de Lenguas Extranjeras (DLE), la entidad responsable de ofrecer la asignatura general de "lengua extranjera" presente en la malla curricular de todas las carreras universitarias.

Desde la década de los años 1960, el DLE ofrece esta asignatura en siete lenguas (alemán, francés, inglés, italiano, mandarín, portugués y ruso). Además de las formaciones generales, el DLE brinda formaciones para docentes de lengua, a nivel de licenciatura, y a nivel de maestría. En 2012, El DLE creó un comité de "Lenguas por Objetivos Específicos" (LOE en adelante) para responder a solicitudes de elaboración de propuestas curriculares del aprendizaje de LOE, y hasta la fecha se ha respondido a solicitudes de varios departamentos del $\mathrm{CU}$ y de varios centros regionales.

Finalmente, como producto de la presente investigación, se desarrolló y se gestiona actualmente una propuesta curricular a partir de 2019 en el centro regional CURNO, ubicado desde 1997 en la ciudad de Juticalpa, Departamento de Olancho. El centro cuenta con una población estudiantil de más de 1,400 estudiantes y forma sobre todo profesionales en las áreas muy específicas de administración empresarial, gestión de negocios y comercio internacional, e ingeniería agroindustrial.

\section{Problemática- Justificación de la Investigación}

En este contexto académico, se ha identificado la siguiente problemática en relación al nivel y la calidad de los cursos generales de inglés I, II y III e Inglés Técnico I y II, no solamente los que se sirven a los 6,000 estudiantes en el CU de la UNAH, en cada período académico, sino también a los 1,400 estudiantes del CURNO: al estar estructurados con base en programas que pretenden transmitir un sentido comunicativo muy general de la lengua, además de ser poco motivadores y muy poco pertinentes para aprender a comunicar en inglés, estos cursos, no permiten desarrollar las habilidades propias y oportunas de los estudiantes, para poder escuchar, leer, escribir y comunicarse en su campo especifico del conocimiento y entorno socio-cultural en inglés. 
Ante esta necesidad de un aprendizaje más profesionalizante- más funcional- del inglés, y como respuesta a la nueva política de reformas educativas de la $\mathrm{UNAH}^{2}$, el comité de LOE del DLE se ha propuesto desarrollar un proyecto de investigación en torno a las modalidades de implementación de un programa de inglés ESP, con el propósito de poder ofrecer cursos, con base en un sentido específico del ámbito de la ciencia de los negocios internacionales. Estos cursos deberán capacitar al futuro profesional en negocios internacionales, con los conocimientos y habilidades requeridos en el escenario mundial, y ser empleados con capacidades negociadoras en empresas nacionales e internacionales del rubro de la administración y comercio internacional en esta región hondureña.

Este proyecto también responde a las necesidades comunicativas e interculturales dentro de un contexto mundial en el que el idioma inglés es una de las principales lenguas que debe saber manejar un negociador a nivel internacional, ya que se utiliza en la mayoría de los países del mundo, de tal forma Bracaj (2014) y Rogerson-Revell (2007), indican que la "necesidad de una lengua franca de negocios ha llevado a la demanda mundial de inglés para un propósito específico".

En concreto, el estudio tiene como fin encontrar las respuestas a la siguiente pregunta de investigación:

¿Cuáles son las necesidades comunicativo-lingüísticas en cuanto a la comprensión oral, la expresión oral, la comprensión escrita y la expresión escrita, así como en cuanto a los estilos, estrategias de aprendizaje y necesidades socio-profesionales o interculturales que manifiestan los estudiantes, docentes y profesionales egresados de las Carreras de Administración de Empresas y Comercio Internacional del CURNO?

\section{Referentes teóricos}

Como enfoque didáctico que nace en los años 1950-1960 (Portine, 1990) como una rama específica dentro de la didáctica de lenguas y culturas, la didáctica de "lenguas por objetivos específicos " (LOE) y específicamente la didáctica del "inglés por objetivos específicos" (ESP -English for Specific Purposes), o IFE, el "inglés con fines específicos"3, surge a partir de un proceso de investigación didáctica para tomar más en consideración las necesidades e intereses del alumno que estudia la lengua extranjera, y cuyo objetivo es adquirir un capital cultural y lingüístico que le sea útil ahora y en el futuro para solucionar problemas relacionados con su ámbito profesional.

Dentro de este contexto, el término specific purposes (objetivos específicos) se refiere a los objetivos comunicativos específicos por alcanzar por el estudiante, en su rol de futuro profesional, dentro de las aptitudes comunicativas de CO (comprensión oral), EO (expresión ora), CE (comprensión escrita) y/o EE (expresión escrita), en el marco de la realización de tareas profesionales especializadas (Eurin, 2000). De la misma manera, el término "específicos" se refiere también a "la lengua que se enseña" (Eurin, 2000): el léxico, la sintaxis y los discursos especializados, y que es la lengua utilizada por profesionales o especialistas en su área de trabajo dentro de un "campo de referencia extra-lingüístico" especifico como la agronomía, la economía, la medicina, el comercio, el turismo, etc.

2 El Modelo Educativo de la UNAH plantea como principios la pertinencia, la calidad y la internacionalización del currículo, (Vicerrectoría Académica, Comité Técnico de Apoyo, 2009).

3 Como enfoque didáctico y de acuerdo a los objetivos instructivos para los cuales la enseñanza es concebida y estructurada, el IFE se subdivide en dos grandes áreas: el "Inglés con Fines Académicos" (IFA) y el "Inglés con Fines Ocupacionales" (IFO), las que a la vez pueden descomponerse en otros campos más específicos en correspondencia con la disciplina u ocupación laboral hacia la que se orienta, (Dudley-Evans \& St. John, 1998). 
Vol. 7 | Investigación Cualitativa en Educación: Avances y Desafíos

En cuanto a la evolución metodológica de la didáctica LOE, esta didáctica evolucionó, tal como la didáctica de lenguas y culturas en general (o "por objetivos generales") de una focalización exclusiva en un proceso de enseñanza-aprendizaje basado en la memorización de listas de vocabulario de especialidad en los años 1950, época de Français Fondamental y de Basic English ${ }^{4}$ ) hacia un aprendizaje con base en listas de vocabulario y expresiones dentro de un campo específico de referencia (p.ej. la agronomía), generadas por "intenciones comunicativas". Este nuevo paradigma fue producto de un análisis de los públicos y sus necesidades de aprendizaje, tal como lo promocionó el referencial "Nivel Umbral" (Coste et al., 1976) a finales de los años 1970.

En este sentido, y a partir de los años 1980, esta transición, en el ámbito de la LOE, se efectuó dentro del marco de discursos de especialidad. Cabe destacar aquí el rol determinante del concepto de "discurso" como "lugar de actualización de la lengua,... de construcción del sentido" (Eurin, 2000), y del "análisis del discurso" como corriente metodológico que estudia el discurso oral y escrito... "como guía para el docente en la selección de documentos para la clase de lengua" (Darot, 1989). El análisis del discurso forma parte de una "lingüística del discurso, aplicada a las problemáticas de investigación en el campo de la enseñanza de lenguas, cuyas bases teóricas y descriptivas fueron propuestas por Peytard \& Moirand (1992), citados por Blanchet \& Chardenet (2011). En este contexto aparece, desde los años 1970, el uso del "documento auténtico", es decir del documento elaborado con fines de comunicación real, y no con fines de enseñanza, para este estudio del discurso oral y escrito. Dentro de la didáctica de LOE, el concepto de discurso constituye por ende la base para la elaboración de matrices y operaciones discursivas, en los textos dichos de especialidad, caracterizados por una "tipología discursiva" específica.

En conclusión, la didáctica de LOE, y por ende de ESP, puede ser calificada como una "didáctica especializada" (Richer, 2008), por su objeto de enseñanza-aprendizaje, que lo constituyen los discursos de especialidad, y por sus objetivos de enseñanza-aprendizaje en relación con tareas profesionales y con base en objetivos comunicativos "específicos" desarrollados en ciertas aptitudes: en $\mathrm{CO}$ o en $\mathrm{CE}, \ldots$ y en ciertos tipos de textos. Además, desempeña un rol fundamental, y más importante que en la didáctica "general" de lenguas, el análisis de necesidades, tanto lingüísticas como culturales, de los aprendices, dentro de situaciones de comunicación profesional específicas. En la metodología de LOE, las necesidades socioprofesionales e interculturales son analizadas en función de determinar y delimitar las situaciones de comunicación dentro de las cuales se desenvolverán los estudiantes en la lengua meta. En este sentido, el conceptor de programas LOE debe buscar respuesta a preguntas de tipo: ¿Con quién hablará el estudiante? ¿Dentro de qué contexto? ¿Qué deberá decir? ¿Qué deberá escuchar? ¿Qué deberá comprender?, etc. (Carras et al., 2007).

\section{Metodología: Procedimiento de la investigación}

Se recurrió a un enfoque mixto de investigación, pero principalmente cualitativo. Se establecieron, con base en el objetivo de la investigación, dos categorías básicas de análisis: por una parte, las aptitudes comunicativas- lingüísticas académicas, profesionales e interculturales en $\mathrm{CE}, \mathrm{CO}$, EE y EO en inglés con fines específicos para los negocios y por otra parte los estilos y estrategias de aprendizaje y evaluación.

\footnotetext{
${ }^{4}$ Instrumentos referenciales que consistían en listas del vocabulario más frecuente de carácter general, respectivamente en francés (Français Fondemental) y de inglés (Basic English).
} 
En calidad de poblaciones informantes participaron los cuatro actores fundamentales para un estudio de esta naturaleza: los estudiantes actuales y egresados, los docentes, y los empresarios-empleadores. Concretamente se examinaron: 100 estudiantes, que representan el $21 \%$ de los estudiantes matriculados actualmente en las Carreras de Administración de Empresas y Comercio Internacional del CURNO. Del mismo modo, se consideraron el $100 \%$ o la totalidad de docentes (33), que laboran actualmente en las mencionadas carreras. También se identificaron un grupo de 39 egresados de una totalidad de 395, y 40 empresarios - empleadores de compañías aledañas que emplean a los egresados de dichas carreras. Para todas las poblaciones se realizó un muestreo a conveniencia y/o intencionado.

Específicamente, para obtener los discursos de dichas poblaciones, abordadas en momentos y espacios diferentes, se validaron teóricamente e implementaron, como instrumentos de recolección de datos:

- Una entrevista de carácter individual y grupal a egresados, docentes y empleadores. Dicha entrevista proporcionó respuestas confiables para el tratamiento de la categoría de análisis respecto a las aptitudes comunicativo- lingüísticas específicas de CE, CO, EE y EO.

- Un cuestionario con preguntas cerradas y abiertas, aplicado únicamente a los estudiantes. Algunas de estas preguntas fueron luego discutidas oralmente entre el equipo de investigación y los informantes. Mediante este instrumento se recogieron respuestas que alimentaron el proceso de interpretación de las dos categorías de análisis del estudio.

- Un espacio de observación participante (Minayo \& Costa, 2019), aplicada únicamente a los empleados y egresados profesionales: mediante una guía de observación, se observó puntualmente y se registró rigorosamente el grado de implementación y ejecución de las aptitudes comunicativo-lingüísticas de los profesionales en inglés, y sus relaciones en el seno de la empresa. Es necesario rescatar la importancia de esta técnica de investigación, en cuanto a la exploración de respuestas a la pregunta de investigación, por su inserción directa en la práctica comunicativa lingüística profesional real, en inglés, de los actores involucrados.

Para lograr interpretar el extenso corpus de datos y encontrar respuesta a la pregunta de investigación planteada, se implementó acertadamente el análisis del discurso desde las opiniones vertidas por los empleadores, profesionales y estudiantes en primer lugar y por parte de los docentes expertos en los contenidos disciplinares, así como de los docentes expertos en la didáctica del inglés, en segundo lugar. Consecuentemente, se procedió al proceso de ordenación y transcripción de los discursos, seguido por la etapa de codificación y organización de los mismos por población y por aptitudes lingüísticas (CE, CO, EO y EE); el proceso concluyó con el análisis, interpretación y concreción en tablas de datos concluyentes de las categorías de análisis observadas, como respuestas válidas y confiables a la pregunta de investigación.

En resumen, metodológicamente el estudio se llevó a cabo rigurosamente en la etapa diagnóstica, es decir de aplicación de instrumentos para la recolección, codificación, graficación, análisis e interpretación de datos cuali-cuantitativos con base en referentes teóricos como Mangiante \& Parpette (2004); referente al análisis e interpretación profunda de datos cualitativos, se fundamentó igualmente en los preceptos de Minayo \& Costa (2019) en cuanto a la necesidad de ordenación, organización, categorización de unidades de análisis e interpretación de datos. 


\section{Resultados}

\subsection{Entrevista Individual con Empleadores de Egresados}

La entrevista fue realizada con 13 empleadores de las empresas de la ciudad de Juticalpa, sede del CURNO, provenientes principalmente de tres áreas: el sector agroindustrial exportador, el sector servicios financieros y el sector educativo técnico. Con esta población se lograron medir las dos categorías de análisis comprometidas para este tipo de estudio, y recomendadas por los autores Cabré (2010) y Mangiante (2007): dominio del inglés y otros idiomas, necesidad del inglés en el desempeño profesional en la empresa, formación de competencias comunicativas profesionales en cuanto a $\mathrm{CO}$, $\mathrm{CE}$, EO y EE, entre otras.

La transcripción, organización y categorización de los discursos de los empleadores evidenciaron que existe la necesidad de que el personal de las empresas se perfeccione en el idioma inglés, a través de la gestión de un programa especializado, el cual tendrá que desarrollar múltiples competencias comunicativo-lingüísticas propias del campo laboral específico (Carras, et al., 2007).

De manera más concreta, en cuanto a las necesidades comunicativo-lingüísticas, los entrevistados manifestaron la ambición que sus empleados sean más capaces de desempeñar, en inglés, tareas comunicativo-lingüísticas de, entre otras:

- CO: de charlas, exposiciones, ... comerciales en eventos nacionales e internacionales.

-EO (interactiva): de negociaciones comerciales presenciales y/o virtuales, de presentación de nuevos productos, de recibimiento e intercambio con socios extranjeros.

= CE y EE: de todo tipo de documentos de carácter comercial.

\subsection{Entrevista Grupal y Cuestionario Dialogado con Estudiantes y Egresados}

Los datos recogidos por este instrumento se sometieron al mismo proceso de tratamiento metodológico anterior y estaban relacionados con las dos categorías en discusión. En suma, se pudo comprobar que, en relación a la primera categoría de análisis la mayoría (90\%) de los (39) egresados y (100) estudiantes encuestados no manejan el inglés, ni tampoco otros idiomas, a un nivel esperado de $\mathrm{A} 2^{5}$ según el Marco Común Europeo de Referencia para las Lenguas (Consejo de Europa, 2001). También un grupo menor de estudiantes manifestó haber usado, sin mucho éxito, el inglés al momento de viajar a otro país. Sin embargo, un porcentaje (10\%) de egresados manifestó poder realizar una conversación inicial a un nivel A1.

\footnotetext{
${ }^{5}$ Referentes de nivel de lengua: $A 1$ - Usuario básico. A2 - Usuario básico. B1 - Usuario independiente, intermedio. B2 -Usuario independiente, intermedio avanzado. Marco Común de Referencia para las lenguas (Consejo de Europa, 2001).
} 
En cuanto a la segunda categoría de análisis, según las opiniones expresadas por la mayoría (98\%) de los estudiantes y egresados, se impone la necesidad de implementación de un programa de inglés más pertinente y avanzado (a nivel B1- B2) que permita poder redactar informes, presentar proyectos, realizar estudios de postgrados en inglés, investigaciones, negociaciones comerciales, entre otras competencias comunicativo-lingüísticas demandadas por los empleadores de las empresas, (véase en apartado 6.1.)

\subsection{Entrevista Grupal Realizada con Docentes de Inglés, Docentes de Contenido Disciplinar y con Autoridades de Gestión Académica}

Se desarrolló una entrevista grupal, entre los cuatro integrantes del equipo de investigación, y dos directores, tres docentes de inglés y cuatro docentes de contenido de la carrera de Comercio Internacional y Administración de Empresas del CURNO. Los datos recogidos estaban relacionados con las dos categorías de análisis discutidas con las poblaciones anteriores.

Los informantes de esta actividad no solamente enfatizaron la importancia de un mayor conocimiento del inglés en vista de las nuevas y mejores oportunidades de empleo y de estudios en el extranjero, sino también la mayor oportunidad y competitividad laboral, y además las oportunidades de conocer otras culturas.

En cuanto a la metodología de enseñanza-aprendizaje en ESP, relacionada con la segunda categoría de análisis, los docentes de ambos grupos consensuaron que un aprendizaje más eficaz y funcional del inglés ESP depende de la implementación de una pedagogía más activa que tome en cuenta los estilos de aprendizaje exitosos, como la realización de tareas en grupos y parejas; el uso de proyectos de clase; de material auténtico en lecturas (Cabré, 2010), de diálogos interactivos, de redacción de textos de diferentes tipología; así como un intenso y pertinente uso de las nuevas tecnologías (TICs).

\subsection{Observación Participante de Empleados, Empleadores y Profesionales Egresados en el Puesto de Trabajo.}

Esta actividad se llevó a cabo con una muestra intencionada de cinco empresas, del rubro financiero y agroindustrial, que realizan actividades comerciales a nivel internacional de carácter de importación y de exportación de la región de Olancho.

En relación a la categoría de análisis referente a las aptitudes comunicativo-lingüísticas en inglés y mediante una guía de observación (Minayo \& Costa, 2019), dos miembros del equipo de investigadores lograron observar ocho empleadores y empleados de dichas empresas (todos ellos profesionales graduados del CURNO), para luego consensuar y registrar que tales empleados, con el fin de establecer negocios de importación y exportación, se enfrentan a la necesidad de realizar tareas comunicativolingüísticas específicas de CE y EE así como de CO y EO. (Véase tabla 1.)

Tabla 1. Algunos ejemplos de tareas comunicativo- lingüísticas en inglés

\begin{tabular}{cc}
\hline CE & EE \\
\hline
\end{tabular}

-Lectura de informes, contratos, ofertas, pólizas, inventarios, entre otras.

-Lectura de manuales de instrucciones de

funcionamiento de equipos y documentos comerciales

-Lectura de correspondencia en inglés ( física y

ofertas, pólizas, inventarios, facturas.

-Redacción de correspondencia, correos virtual)

y cartas comerciales (física y virtual) 


\begin{tabular}{ll}
\hline \multicolumn{1}{c}{ CO } & \multicolumn{1}{c}{ EO } \\
\hline -Comprensión de llamadas internacionales & -Realización de llamadas \\
-Recibimiento de visitantes internacionales & internacionales \\
-Realización de encuentros vía Skype con & -Ejecución de exposiciones orales de \\
empresarios y representantes extranjeros & informes \\
& -Intervención en proyectos de campo \\
\hline
\end{tabular}

Finalmente, en relación con la implementación de la competencia intercultural (Consejo de Europa, 2001), el equipo investigador pudo determinar la necesidad de asistir y participar, de manera cross-culturalmente clara y apropiada, con contrapartes internacionales en investigaciones científicas, en reuniones de trabajo, de estudios de mercado, en seminarios, talleres, conferencias nacionales y principalmente internacionales.

\section{Conclusiones}

En correspondencia con el objetivo principal del estudio, se lograron determinar de manera exitosa, a través de un proceso riguroso de análisis e interpretación de los discursos de las poblaciones sujetas del estudio en las entrevistas y en la observación participante, las necesidades comunicativo-lingüísticas específicas en inglés, por una parte, y las necesidades de formación en cuanto a las competencias comunicativolingüísticas profesionales y académicas disciplinares en CO y EO, CE y EE en inglés, por otra parte (véase capítulo 6). Son precisamente estas necesidades que deberán de ser desarrolladas en una posterior propuesta curricular ESP.

En síntesis, en cuanto a los resultados referentes a las aptitudes comunicativolingüísticas en inglés, resulta claro que se ha manifestado una importante necesidad de un mayor dominio, y un dominio más funcional y profesional en esta lengua, en las cuatro aptitudes señaladas, de parte de los actores principales en este estudio: los estudiantes y empleados del rubro financiero y agroindustrial mencionados. Esta necesidad no solamente se identificó claramente en las diferentes entrevistas realizadas con todos los actores, pero fue ratificada y comprobada (más) directamente en el proceso de observación participante, durante el cual se lograron identificar con mayor precisión las tareas comunicativo-lingüísticas que necesitan poder realizar los estudiantes y empleados.

En cuanto a los resultados en relación a los estilos-estrategias de aprendizaje, se ha evidenciado, en las diferentes entrevistas, que, en concordancia con las necesidades de formación expresadas por los estudiantes y empleados por una parte, y por los docentes por otra parte, que los docentes responsables del futuro programa ESP necesitarán poder manejar una pedagogía diferenciada en acorde con los lineamientos y principios de una pedagogía activa en la cual se tomará más en cuenta la motivación y los diferentes estilos de aprendizaje de los estudiantes en proceso de formación. ${ }^{6}$ Por otra parte, este programa ESP deberá estar basado en la metodología más actualizada con base en proyectos, llamada "enfoque accional". Mediante esta metodología se visualiza la elaboración de proyectos de clase, lo que implica una participación más activa de los estudiantes, "actores sociales" también fuera del aula, ${ }^{7}$ en una secuencia de actividades de CO, EO, CE y/o EE.

\footnotetext{
${ }^{6}$ Realizando tanto actividades correspondientes a estudiantes de tipo "visual" como a estudiantes de tipo "auditivo", "táctil" o "kinésico" (Gardner, 1993)

7 Trabajando en grupos y parejas; leyendo, escuchando y redactando documentos auténticos del ámbito comercial; conversando sobre actividades cotidianas, académicas y profesionales; etc.
} 
En este sentido se está visualizando otra línea interesante de investigación que se enfocaría en las representaciones sociales de los docentes de lengua acerca de la didáctica diferenciada ESP para los negocios. Será precisamente la simbiosis entre el diagnóstico de las necesidades de formación lingüística y la inclusión de estas representaciones sociales, que servirá de base para el éxito de una propuesta de reforma curricular en lo que respecta a la enseñanza del inglés en la UNAH.

En suma, gracias a este proyecto, se podrá acceder al desarrollo de un continuo con mayores y exitosas ventajas académicas y profesionales para los estudiantes y egresados de dichas Carreras y eventualmente otras disciplinas del CURNO, con el fin máximo de potencializar la calidad y la internacionalización de la educación de la UNAH.

\section{Referencias}

Basturkmen, H. (2010). Developing Courses in English for Specific Purposes. Basingstoke, UK : Palgrave Macmillan.

Blanchet, P. \& Chardenet, P. (2011). Guide pour la recherche en didactique des langues et des cultures. Agence Universitaire de la Francophonie. Paris

Bracaj, M. (2014). "Teaching English for Specific Purposes and teacher training". European Scientific Journal, 10(2).

Cabré, M-T (2010). "Recursos lingüísticos en la enseñanza de lenguas de especialidad". Instituto Universitario de Lingüística Aplicada. UniversitatPompeuFabra(Barcelona). Obtenida el 3 de Marzo de 2017, de http://cvc.cervantes.es/obref/aeter/conferencias/cabre.htm

Carras C., Tolas J., Kohler P. \& Szilagyi E., (2007). Le Français sur Objectifs Spécifiques et la classe de langue. Paris. CLE international.

Consejo de Europa (2001). Un Marco Común de Referencia para las lenguas: aprender, enseñar, evaluar. Strasbourg. Didier.

Coste,D. et al., (1976). Un niveau seuil. Consejo de Europa. Strasbourg.

Darot, M. (1989) "A quoi sert l'analyse du discours", in Reflet No.31.Paris : Association Reflet.

Dudley-Evans, T., \& St John, M. J. (1998). Developments in English for specific purposes: A multidisciplinary approach. Cambridge University Press.

Eurin, S. (2000) Français sur Objectifs Spécifiques. Cours de Maitrise. Université Stendhal de Grenoble 3. France

Gardner, H. (1993). Inteligencias múltiples. Barcelona : Ed. Paidós.

Hutchinson, T. \& Waters, A. (1987) English for Specific Purposes. A Learning Centred Approach. Cambridge: Cambridge University Press.

Mangiante, J-M. \& Parpette C., (2004). Le Français sur Objectifs Spécifiques : de I'analyse des besoins à l'élaboration d'un cours. Paris. Hachette FLE.

Mangiante, J-M. (2007). "Une démarche de référentialisation en français des professions : le partenariat universités - Chambre de Commerce et d'Industrie de Paris" (CCIP). Université d'Artois. Disponible en : http://www.fdlm.org/fle/ra/articles/modele.php

Minayo, M., \& Costa, A. (2019). Técnicas que hacen uso de la palabra, de la observación y de la empatía. Aveiro. Portugal : Ludomedia.

Portine, H. (1990) «Les langues de spécialité comme enjeux de représentations » Le Français dans le Monde, Recherches et Applications. Apprentissages et usage des langues dans le cadre européen, Hachette, Paris.

Richer, J.J. (2008) «Le francais sur objectifs spécifiques (FOS): une didactique spécialisée » Synergies Chine No.3. Gerflint, Paris.

Rogerson-Revell, P. (2007). "Using English for international business: A European case study". English for specific purposes, 26(1), 103-120.

Vicerrectoría Académica, Comité Técnico de Apoyo, UNAH (2009). Modelo Educativo de la UNAH. Ciudad Universitaria. Serie de Publicaciones de la Reforma Universitaria No 3. 